OPEN ACCESS

Edited by:

Joseph Edward Hayward, Juravinski Cancer Centre, Canada

Reviwed by:

Mehmet Burcin Unlu, Boğaziçi University, Turkey

Kai Yin,

Central South University, China

*Correspondence: Qiyin Fang

qiyin.fang@mcmaster.ca Steve Guofang Shen

shengf@sumhs.edu.cn

tThese authors have contributed equally to this work

Received: 31 August 2021 Accepted: 23 November 2021 Published: 22 December 2021

Citation:

Zhang W, Alghannam F, Zhu Y, Zhang J, Wohl GR, Haugen HK, Qin Z, Xie G, Fang $Q$ and Shen SG (2021) Effects of Drilling Technology on MiniImplant Primary Stability: A Comparison of the Mechanical Drilling and Femtosecond Laser Ablation. Front. Phys. 9:766644.

doi: 10.3389/fphy.2021.766644

\section{Effects of Drilling Technology on Mini-Implant Primary Stability: A Comparison of the Mechanical Drilling and Femtosecond Laser Ablation}

\begin{abstract}
Wenbin Zhang ${ }^{1 \dagger}$, Fahad Alghannam ${ }^{2,3+}$, Yingchao Zhu ${ }^{1}$, Jianfei Zhang ${ }^{1 \dagger}$, Gregory R Wohl ${ }^{3,4}$ Harold K. Haugen ${ }^{5,6}$, Zhipeng Qin ${ }^{1}$, Guoqiang Xie ${ }^{1}$, Qiyin Fang ${ }^{3,5 *}$ and Steve Guofang Shen ${ }^{7 \star}$

${ }^{1}$ Department of Oral and Craniomaxillofacial Surgery, Ninth People's Hospital, Shanghai Jiaotong University School of Medicine, National Clinical Research Center for Oral Diseases, Shanghai, China, ${ }^{2}$ National Center for Nanotechnology and Semiconductor, King Abdulaziz City for Science and Technology (KACST), Riyadh, Saudi Arabia, ${ }^{3}$ School of Biomedical Engineering, McMaster University, Hamilton, ON, Canada, ${ }^{4}$ Department of Mechanical Engineering, McMaster University, Hamilton, ON, Canada, ${ }^{5}$ Department of Engineering Physics, McMaster University, Hamilton, ON, Canada, ${ }^{6}$ Department of Physics and Astronomy, McMaster University, Hamilton, ON, Canada, ${ }^{7}$ Shanghai University of Medicine and Health Sciences, Shanghai, China
\end{abstract}

Objectives: Primary stability is a fundamental prerequisite in predicting the prognosis of a mini-implant (MI) as a skeletal anchorage. This study aims to evaluate the influence of implant site preparation technology on the primary stability of Ml.

Methods: A total of 108 bovine cortical bone samples were fabricated to three thicknesses $(0.5,1.0$, and $1.5 \mathrm{~mm})$. For each thickness group, the samples were divided into three subgroups: I (without site preparation), II (site preparation with a mechanical drill), and III (site preparation with femtosecond laser ablation). After MI insertion into these samples, the pull out strength of Ml was measured by lateral pull out tests.

Results: For the $0.5 \mathrm{~mm}$ thickness samples, the lateral pull-out strength was $9.9 \pm 2.7 \mathrm{~N}$ in subgroup I, $6.7 \pm 2.1 \mathrm{~N}$ in subgroup II, and $15.2 \pm 2.6 \mathrm{~N}$ in subgroup III. For the $1.0 \mathrm{~mm}$ thickness samples, the lateral pull-out strength was $39.3 \pm 2.5 \mathrm{~N}$ in subgroup I, $38.2 \pm 2.7 \mathrm{~N}$ in subgroup II, and $46.3 \pm 1.7 \mathrm{~N}$ in subgroup III. For the $1.5 \mathrm{~mm}$ thickness samples, the lateral pull-out strength was $73.9 \pm 4.8 \mathrm{~N}$ in subgroup I, $70.1 \pm 2.8 \mathrm{~N}$ in subgroup II, $75.0 \pm 2.2 \mathrm{~N}$ in subgroup III. No signs of carbonization or substantial cracking were visible in any of the bone samples.

Conclusion: Site preparation with laser ablation significantly improved the lateral pull-out strength over mechanical preparation and control (no site preparation) in thinner cortical bone samples (1.0 and $0.5 \mathrm{~mm}$ ). Such improvement in lateral pull-out strength decreases as the samples become thicker and diminishes in thick $(1.5 \mathrm{~mm})$ cortical bone samples.

Keywords: mini-implant, primary stability, laser ablation, femtosecond laser, site preparation

Abbreviations: BS, beam-splitter; CCD, charge-coupled device; MI, mini-implant; PD, photodiode. 


\section{INTRODUCTION}

A mini-implant (MI) is a device smaller than a conventional dental implant that provides skeletal anchorage for orthodontic tooth movement. As the MI is directly attached to the bony appendages, it provides an absolute anchorage with an ideal movement of the targeted teeth. The advantages of a MI over a dental implant include less invasiveness, relative comfort, and fewer limitations in the patient [1]. Hence, the use of MI application as an anchorage is favoured by the orthodontists and patients. However, the success rate of MI is lower than those of dental implants [2]. Therefore, it's essential to find the affecting factors of the MI success rate [3].

A number of studies on surrounding bone volume and unique MI design suggested that primary stability was critical in ensuring the success of MI [4]. In addition, the drilling protocols for implant site preparation may be a factor in achieving robust primary stability [5], while the effect of implant site preparation technology on primary stability is not thoroughly studied [6].

At present, implant site preparation was typically achieved by mechanical drilling in clinical practices.

High intensity lasers have been widely used in machining applications in the past decade. Recently, ultrafast laser with picosecond to femtosecond pulses have been demonstrated with unique material processing capabilities that can produce novel structures in the micro to nanoscale [24, 25]. In the case of surgical applications, short ( $<$ nanosecond) pulse laser ablation, a non-contact technique usually associated with less thermal damage, is an alternative technology for MI site preparation. Lo et al. demonstrated accelerated bone healing after femtosecond laser ablation in a calvarial defect model [7]. Baek et al. compared the cut surface of the laser osteotomy with conventional mechanical osteotomy [8]. They found biologically open-cut surfaces in the laser osteotomy samples and a flattened smear layer in piezoelectric osteotomy samples. These results explained why laser osteotomy increases bone healing compared with conventional mechanical bone cutting. Femtosecond laser ablation technology was used for osteoodonto-keratoprosthesis procedures by Berget et al. [9], who showed that the laser cut holes were as strong as burr drilled holes and with a smoother surface in SEM results. Consequently, laser osteotomy opens up the possibility to custom fit the hole precisely to the implant's width, which represents a potential advantage of the laser over the conventional burr.

The goal of this study was to assess the effect of MI site preparation with a femtosecond laser ablation system on the MI primary stability. Bovine cortical bone samples at different thicknesses were used with MI site preparation performed with a mechanical drill or ultrafast laser ablation, respectively. After MI insertion, the lateral pull-out strength of MI was measured with a tensile testing machine, and the results were compared to a control group without site preparation.

\section{MATERIALS AND METHODS}

\section{Preparation of Bone Samples}

Bovine bones were obtained from a local slaughterhouse. The attached soft tissue and periosteum were removed, and the cortical bones were harvested. Typical bone samples were cut into different thicknesses $(0.5,1,1.5 \mathrm{~mm})$ with a low-speed diamond saw (Diamond Slicing Blades, UKAM Industrial Superhard Tools, Valencia, CA, United States). After sectioning, the bone samples were labeled with the date of harvest and stored in the freezer. The samples in each group were further divided into three subgroups according to the MI site preparation methods. In subgroup I (control), the MI was screwed in bone samples without implant site preparation. In subgroup II, the MI was screwed into samples with a hole drilled by a mechanical drill. In subgroup III, the MI was screwed into samples with a hole drilled by femtosecond laser ablation.

\section{Preparation of Ultrafast Laser Micromachining System}

The ultrafast laser system used was an amplified Ti: Sapphire system (Coherent, Santa Clara, CA, United States) with $3.5 \mathrm{~W}$ average power, $170 \mathrm{fs}$ pulse duration (FWHM), and $1 \mathrm{kHz}$ repetition rate at $800 \mathrm{~nm}$. A plano-convex focusing lens (effective focal length $12.5 \mathrm{~cm}, \mathrm{BK} 7$; Thorlabs, Newton, NJ, United States) was mounted on a single axis translation stage (MFN25PP, Newport, Irvine, CA, United States) for laser focal point adjustment along the $Z$-axis (Figure 1). A detailed description of the system was reported by An et al. [10]. The samples were placed on a holder with an X-Y translation stage. The ablation process was monitored using a confocal monochrome charged-couple device (CCD) camera, and a white LED light source was for illumination purpose.

\section{Site Preparation With a Mechanical Drill}

The MI sites were prepared with a $1.0 \mathrm{~mm}$ diameter drill bit powered by a powered instrument driver (CORE Console, Stryker Inc., MI, United States) at 20,000 rpm. The holes with a $1.0 \mathrm{~mm}$ diameter were created under irrigation with saline solution.

\section{Site Preparation With Laser Ablation}

The samples were ablated in a sealed glass container with a plastic lid [10]. A small circular opening (20 mm diameter) in the lid was covered by a quartz slide ( $0.18 \mathrm{~mm}$ thickness), which acted as a window for the laser beam. The quartz slide was sealed in the lid using a transparent epoxy adhesive. Paraffin wax was used to fix the samples at the bottom of the container. The focal plane of the beam was aligned with the bone surface. A hole with an about $1.0 \mathrm{~mm}$ diameter was created using $15.1 \pm 1.7 \mathrm{~J} / \mathrm{cm}^{2}$ beam fluence and $1 \mathrm{~mm} / \mathrm{s}$ scanning speed. Concentric circle scanning from the center towards the outside and compressed air flow was applied to remove the debris during processing [10]. 


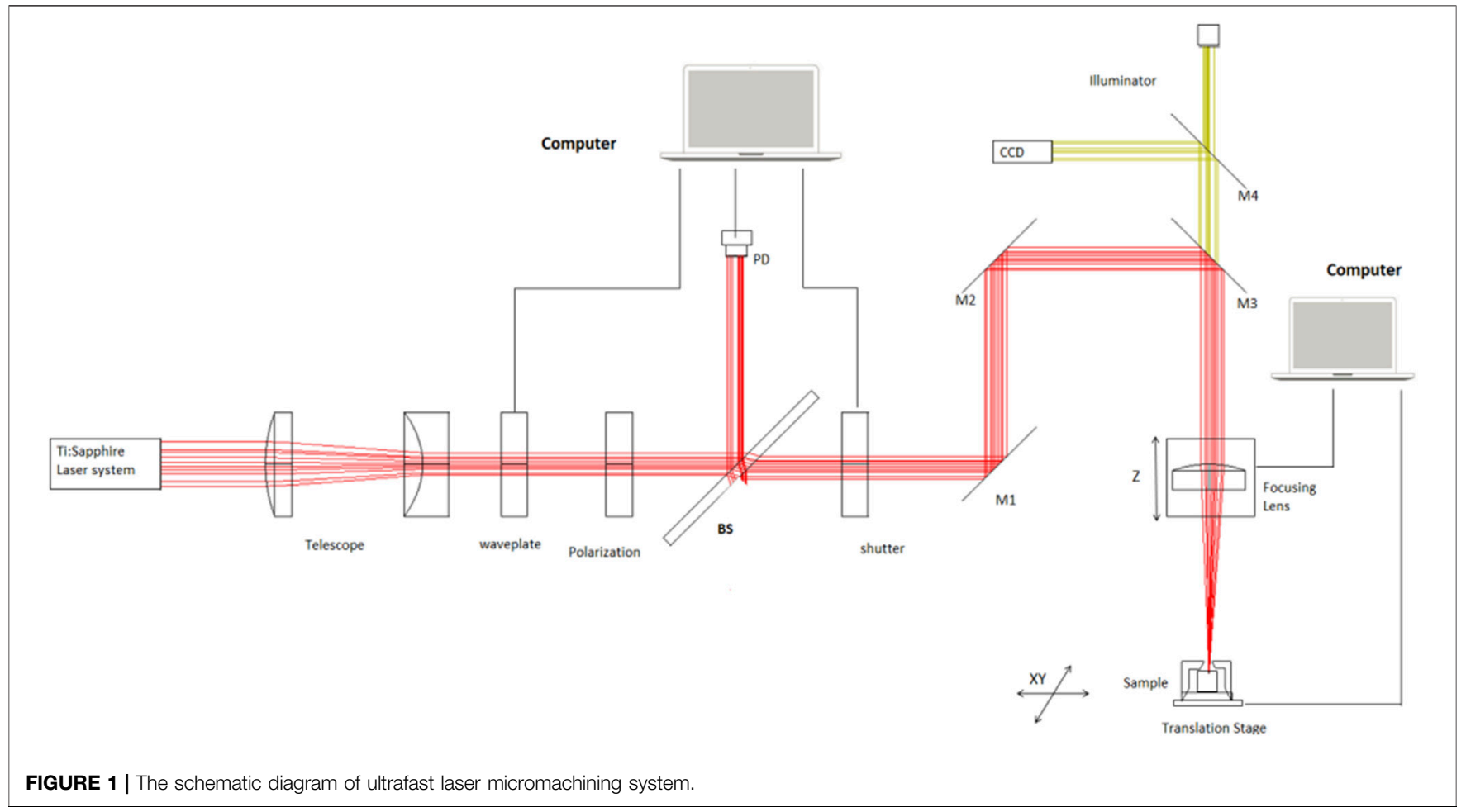

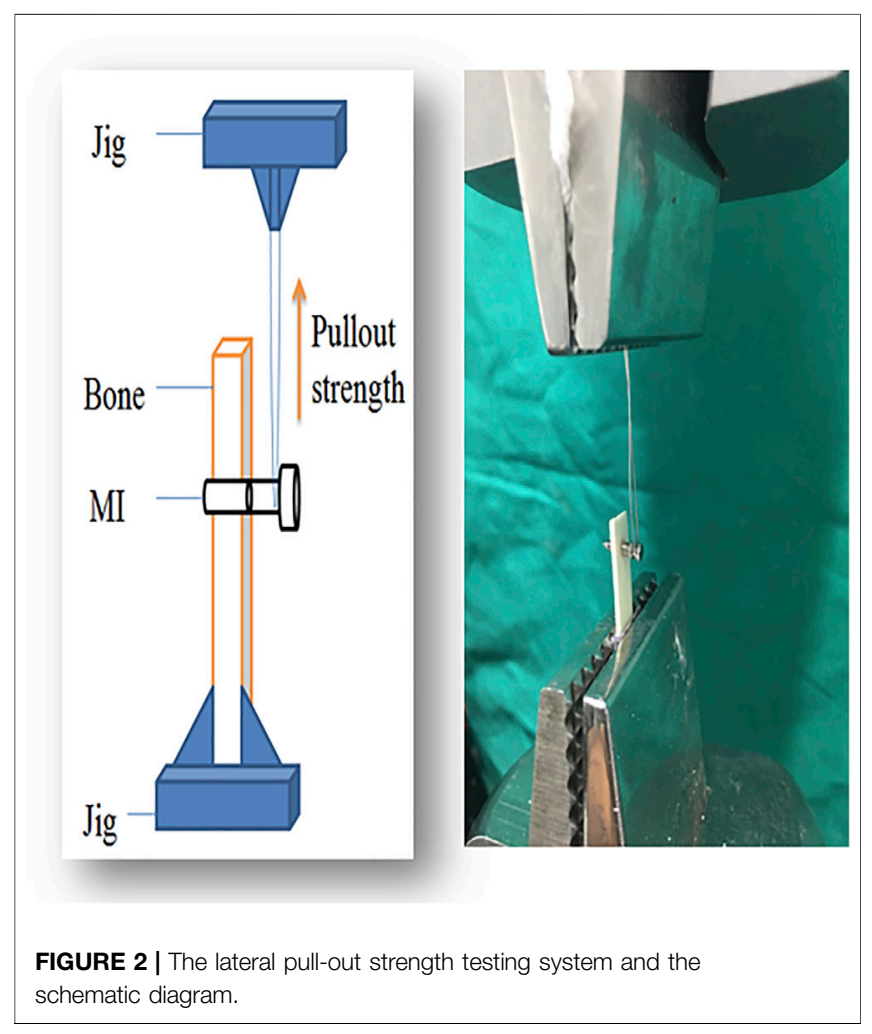

\section{Test of Lateral Pull-Out Strength}

One hundred and eight (108) commercial mini-screw implants (MI), $1.6 \mathrm{~mm}$ in diameter and $9 \mathrm{~mm}$ in length (Anchorage orthodontic screws, Ningbo Cibei Medical Treatment Appliance, China), were used. After MI insertion, the bone samples were transferred and secured in a sample holder in the Instron machine (Instron Corp., Canton, MA, United States) (Figure 2). Lateral pull-out strength testing was performed by fixing an adapter that was custom made to fit a 0.040 -inch stainless steel wire. The steel wire was tightened over the head to provide a firm hold on the MI and attached to the Instron machine. A force of $10 \mathrm{~mm} / \mathrm{min}$ with a maximum of $10 \mathrm{~N}$ was applied perpendicular to the mini-screw long axis until failure occurred. Peak load at failure was recorded in Newton $(\mathrm{N})$. If there was no MI pull-out, a preload with a maximum of $10 \mathrm{~N}$, a minimum of $2 \mathrm{~N}$, and 50 cycles were applied. Then a lateral force was applied at a speed of $10 \mathrm{~mm} / \mathrm{min}$ with a maximum of $100 \mathrm{~N}$, and the peak load at failure was recorded. To reduce the variability in the pull-out strength due to the bone sample variability, $36 \mathrm{MI}$ were used in each group.

\section{Statistical Analysis}

Statistical analysis was carried out with the commercial software SPSS (SPSS, Version 14.0, Chicago, IL). Two-way factorial analysis of variance (ANOVA) was used to assess overall differences between groups, followed by comparisons to evaluate differences between individual groups. A significance level was set at $p<0.05$.

\section{RESULTS}

The surface morphology of the bone sample was analyzed with a scanning electron microscope (SEM). Top-view SEM image of a 


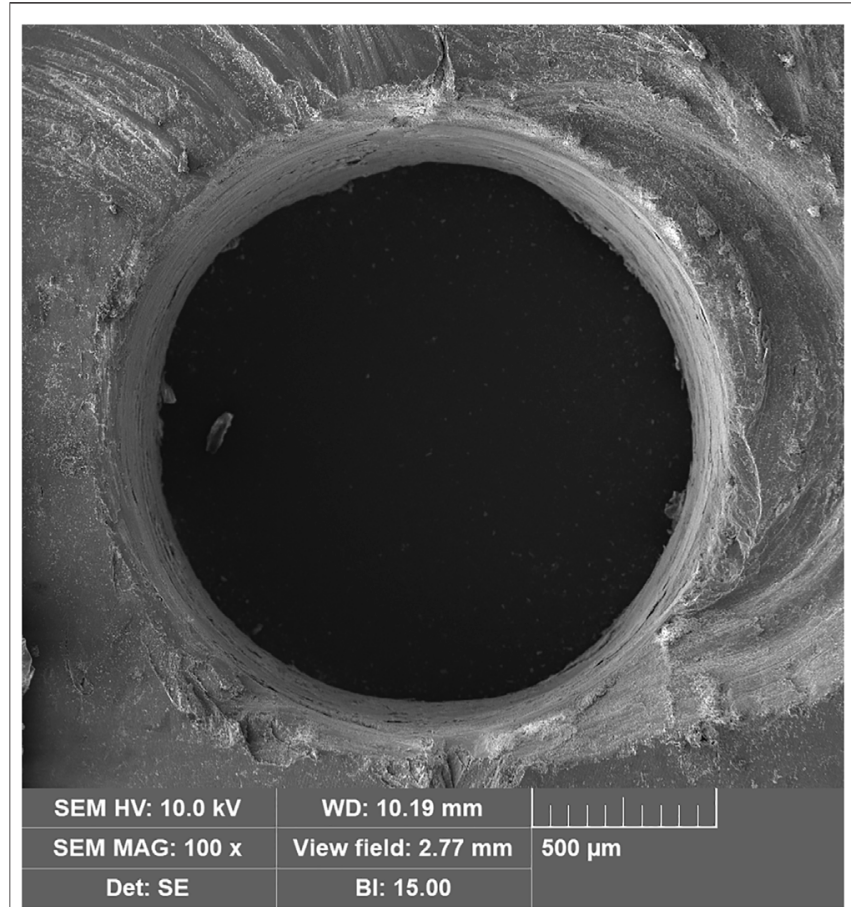

FIGURE 3 | SEM images of Ml site preparation with $1.0 \mathrm{~mm}$ diameter drill bits, 20,000 rpm.

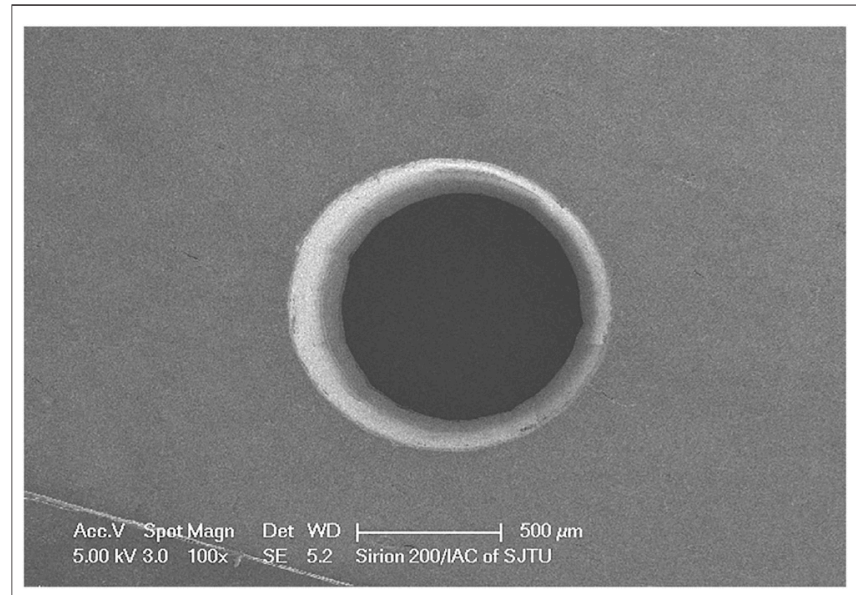

FIGURE 4 | SEM images of Ml site preparation with $3.5 \mathrm{~W}$ average power laser, $170 \mathrm{fs}$ pulse duration (FWHM), $1 \mathrm{kHz}$ repetition rate at $800 \mathrm{~nm}$.

hole prepared with a mechanical drill is shown in Figure 3. The surface characterization showed a cone-shape hole covered and closed by a coating resembling the smear layer. Top-view SEM image of a hole prepared with laser ablation is shown in Figure 4. The surface characterization showed a conical hole without a smear layer. No signs of carbonization or substantial cracking are visible in any of the bone samples. The quantitative lateral pullout strength measurements are listed in Table $\mathbf{1}$.
TABLE 1 | The lateral pull-out strength $(\mathrm{N})$ in three sample thickness groups and subgroups: subgroup I: control, without implant site preparation; subgroup II, with mechanical drill; subgroup III: by femtosecond laser ablation.

\begin{tabular}{lccc}
\hline Thickness $(\mathbf{m m})$ & Subgroup I & Subgroup II & Subgroup III \\
\hline 0.5 & $9.9 \pm 2.7 \mathrm{~N}$ & $6.7 \pm 2.1 \mathrm{~N}$ & $15.2 \pm 2.6 \mathrm{~N}$ \\
1.0 & $39.3 \pm 2.5 \mathrm{~N}$ & $38.2 \pm 2.7 \mathrm{~N}$ & $46.3 \pm 1.7 \mathrm{~N}$ \\
1.5 & $73.9 \pm 4.8 \mathrm{~N}$ & $70.1 \pm 2.8 \mathrm{~N}$ & $75.0 \pm 2.2 \mathrm{~N}$
\end{tabular}

\section{DISCUSSION}

Implant stability is the fundamental requirement for successful skeletal anchorage, and the implant must be maintained for the entire orthodontic period to avoid micro-movements. The stability is obtained from the cortical bones and the remodeling of adjacent bones during the healing process. Delgado et al. found that implant site preparation, corticalbone thickness, and implant design strongly influenced MI's primary stability for orthodontic anchorage [11].

To achieve better MI primary stability, less invasive insertion process and greater thickness cortical bone is recommended. However, cortical bone thickness of the implant site is limited in the middle and distal parts of the upper alveolar processes. Silvestrini et al. reported a mean thickness of the cortical bone in the maxilla of $1.10 \mathrm{~mm}$ buccally and $1.27 \mathrm{~mm}$ on the palatal side [12]. Hourfar et al. found the cortical bone thickness of the anterior palate with values ranging from 0.33 to $1.65 \mathrm{~mm}$ [13]. Mohlhenrich et al. measured bone density with cone-beam computed tomography [14]. They found cortical thickness ranged from 0.49 to $0.98 \mathrm{~mm}$ at 11 different positions in human cadaver maxillae. For testing the primary stability of MI, human cadaveric bone is a less suitable model as the thickness of cortical bone is variable. The variability of the cadaver bone may render it difficult to distinguish the effects between the thicknesses of cortical bone and site preparation technology. In this study, the standardized bovine bone samples were used to evaluate the effects of drilling technology on miniscrew implant's primary stability. The bone samples were divided into respective groups according to the fabricated thickness $(0.5$, $1,1.5 \mathrm{~mm})$.

The insertion torque, periotest, and resonance frequency analysis are used to evaluate the stability of mini-screw implants [15]. From a clinical perspective, the consensus is that the implant should bear the lateral pull-out strength $(10 \mathrm{~N})$, which is the strength used for tooth movement as an anchorage [16]. Therefore, the measurement of MI pull-out strength under loading perpendicular to the MI long-axis was to evaluate MI's primary stability in this study. For simulating the process in actual clinical practice (the MI implant should be able to withstand tens to hundreds of cycles of pull-out), the 50 cycles of repeated lateral pull-out loads $(2-10 \mathrm{~N})$ were performed after initial lateral pull-out strength $(10 \mathrm{~N})$ was introduced. Then the lateral pull-out strength of the MI was measured.

In all three groups, the lateral pull-out strength increased with increasing cortical bone thickness (Table 1). The lateral pull-out strength was higher than $10 \mathrm{~N}$ in the group of cortical bone 
thicknesses of 1 or $1.5 \mathrm{~mm}$, which means it can be used as a skeletal anchorage. However, in the group of cortical bone thicknesses of $0.5 \mathrm{~mm}$, the lateral pull-out strength in the subgroups I and II was less than $10 \mathrm{~N}$. On the other hand, the ultrafast laser prepared subgroup III shows lateral pull-out strength of $15.2 \pm 2.6 \mathrm{~N}$, which demonstrated that such improvement is clinically significant.

The decrease of pull-out strength in thinner samples is due to the lack of stabilizing torque (bending moment) to resist the lateral load applied to the screw head. In such thin samples, only the middle of the MI shaft is held in the bone, and the tip of the MI is unsupported. When the head of the MI is pulled, there is insufficient bone to resist the tipping of the MI. With thicker bone samples, more of the shaft of the MI is supported-providing a greater resistance (greater bending moment) to the lateral load at the head of the screw. The small improvement in lateral pull-out strength for even the thin bone samples demonstrates the importance of the stability of the bone bed. It is also reported that strain at the bone-implant interface rose with a decreasing MI diameter in finite element analysis [17]. Small diameter screws presented an increased risk of fracture [18] and more microcracks [19]. For the conventional MI site preparation with burr drilling, vibration and thermal damage might generate a side effect of microfracture. Hemanth et al. found the MI site preparation using drilling led to the occurrence of microdamage on the bone [20]. The lateral effects were seen around the implant surface and extend up to $1 \mathrm{~mm}$ [21]. These microcracks acted as areas of demineralization or voids and hampered the primary stability of the MI. Extensive microcracks with clear damage were also reported by other authors [22]. Although no signs of carbonization or strong cracking were visible in either of the bone samples, the electron microscopy images showed fewer smears and a smoother surface in laser ablated holes (Figure 4) than in electric drilled hole (Figure 3).

As for MI site preparation with laser ablation, vibration and thermal damage are related to the laser wavelength, pulse duration, and thermal relaxation constant. For a given type of laser-tissue interaction, if the duration of the laser pulse and subsequent ablation process is shorter than the thermal relaxation time of the irradiated volume, the ablation is thermally confined [23]. Our previous study showed that, using optical microscopy and Raman confocal microscopy, the ultrafast laser osteotomy showed no observable thermal damage, carbonization, or microcracks on bovine bone [23].

The result of the present investigation showed that primary stability was significantly influenced by MIs site preparation technology, especially for the site in which cortical bone thickness is less than $1 \mathrm{~mm}$. As this study only evaluated the primary stability of the MIs, further animal studies are required to better understand the bone remodeling and osteointegration in response to the implant site preparation. Finally, clinical trials to

\section{REFERENCES}

1. Liu Y, Yang Z-j, Zhou J, Xiong P, Wang Q, Yang Y, et al. Comparison of Anchorage Efficiency of Orthodontic Mini-Implant and Conventional evaluate the long-term stability of different implant site preparation technologies are essential for making clinical use possible.

\section{CONCLUSION}

In this study, we demonstrated that femtosecond laser ablation is a promising technology for implant site preparation in the thin cortical region (as in the posterior maxilla). Laser ablation prepared samples show significant improvements in MI primary stability, especially in thinner samples where mechanical preparation failed to meet clinical requirements.

\section{DATA AVAILABILITY STATEMENT}

The original contributions presented in the study are included in the article/Supplementary Material, and further inquiries can be directed to the corresponding author.

\section{AUTHOR CONTRIBUTIONS}

WZ, FA, QF, GRW, HKH and SGS contributed to conception and design of the study. YZ and GRW prepared the bone samples. YZ and JZ performed the test of lateral pull out strength. ZQ, and GX prepared the MI site with laser ablation. WZ and FA performed the statistical analysis and wrote the manuscript. All authors contributed to data analysis, manuscript revision and approved the submitted version. WZ, FA, YZ and JZ contributed equally to this study.

\section{FUNDING}

This study was supported in part by the National Key Research and Development Project (Q2017YFGX070045), the Shanghai International Scientific and Technological Project (17410710500, 19441906000), Project of Medical Robots (IMR-NPH202003) from the Clinical Joint Research Center of the Institute of Medical Robots, Shanghai Jiao Tong University-Shanghai Ninth People's Hospital, the Pudong New Area project (PW2016E-1), and the Foshan Science and Technology Innovation Project (2018IT100212).

\section{ACKNOWLEDGMENTS}

The authors gratefully acknowledge Dr. Hai Hu (Department of Orthopaedics; Sixth People's Hospital) for kindly supplying the mechanical test equipment.

2. Kakali L, Alharbi M, Pandis N, Gkantidis N, Kloukos D. Success of Palatal Implants or Mini-Screws Placed Median or Paramedian for the Reinforcement 
of anchorage during Orthodontic Treatment: a Systematic Review. Eur J Orthod (2019) 41:9-20. doi:10.1093/ejo/cjy015

3. Ichinohe M, Motoyoshi M, Inaba M, Uchida Y, Kaneko M, Matsuike R, et al. Risk Factors for Failure of Orthodontic Mini-Screws Placed in the Median Palate. J Oral Sci (2019) 61:13-8. doi:10.2334/josnusd.17-0377

4. Lee J, Jeong Y-H, Pittman J, Deguchi T, Johnston WM, Fields HW, et al. Primary Stability and Viscoelastic Displacement of Mini-Implant System under Loading. Clin Biomech (2017) 41:28-33. doi:10.1016/j.clinbiomech.2016.11.004

5. El-Kholey KE, Elkomy A. Does the Drilling Technique for Implant Site Preparation Enhance Implant success in Low-Density Bone? A Systematic Review. Implant Dent (2019) 28:500-9. doi:10.1097/id.0000000000000917

6. Turkyilmaz I, Tozum TF. Enhancing Primary Implant Stability by Undersizing Implant Site Preparation: A Human Cadaver Study. J Stomatology, Oral Maxillofac Surg (2020) 121:58-62. doi:10.1016/j.jormas.2019.04.016

7. Lo DD, Mackanos MA, Chung MT, Hyun JS, Montoro DT, Grova M, et al. Femtosecond Plasma Mediated Laser Ablation Has Advantages over Mechanical Osteotomy of Cranial Bone. Lasers Surg Med (2012) 44:805-14. doi:10.1002/lsm.22098

8. Baek K-w., Deibel W, Marinov D, Griessen M, Dard M, Bruno A, et al. A Comparative Investigation of Bone Surface after Cutting with Mechanical Tools and Er:YAG Laser. Lasers Surg Med (2015) 47:426-32. doi:10.1002/ lsm.22352

9. Berg BI, Peyer M, Kuske L, Augello M, Schötzau A, Steineck M, et al. Comparison of an Er: YAG Laser Osteotome versus a Conventional Drill for the Use in Osteo- Odonto-keratoprosthesis (OOKP). Lasers Surg Med (2019) 51:531-7. doi:10.1002/lsm.23053

10. An R, Khadar GW, Wilk EI, Emigh B, Haugen HK, Wohl GR, et al. Ultrafast Laser Ablation and Machining Large-Size Structures on Porcine Bone. J Biomed Opt (2013) 18:70504. doi:10.1117/1.JBO.18.7.070504

11. Delgado-Ruiz R, Gold J, Somohano Marquez T, Romanos G. Under-drilling versus Hybrid Osseodensification Technique: Differences in Implant Primary Stability and Bone Density of the Implant Bed walls. Materials (2020) 13:390. doi:10.3390/ma13020390

12. Silvestrini Biavati A, Tecco S, Migliorati M, Festa F, Marzo G, Gherlone E, et al. Three-dimensional Tomographic Mapping Related to Primary Stability and Structural Miniscrew Characteristics. Orthod Craniofac Res (2011) 14:88-99. doi:10.1111/j.1601-6343.2011.01512.x

13. Hourfar J, Kanavakis G, Bister D, Schätzle M, Awad L, Nienkemper M, et al. Three Dimensional Anatomical Exploration of the Anterior Hard Palate at the Level of the Third Ruga for the Placement of Mini-Implants - a Cone-Beam CT Study. Eortho (2015) 37:589-95. doi:10.1093/ejo/cju093

14. Möhlhenrich SC, Heussen N, Winterhalder P, Prescher A, Hölzle F, Modabber A, et al. Predicting Primary Stability of Orthodontic Mini-implants, According to Position, Screw-size, and Bone Quality, in the Maxilla of Aged Patients: a Cadaveric Study. Eur J Oral Sci (2019) 127:462-71. doi:10.1111/eos.12651

15. Nienkemper M, Wilmes B, Panayotidis A, Pauls A, Golubovic V, Schwarz F, et al. Measurement of Mini-Implant Stability Using Resonance Frequency Analysis. Angle Orthod (2013) 83:230-8. doi:10.2319/043012-354.1

16. Valente MLd. C, De Castro DT, Shimano AC, Lepri CP, Dos Reis AC. Analysis of the Influence of Implant Shape on Primary Stability Using the Correlation of
Multiple Methods. Clin Oral Invest (2015) 19:1861-6. doi:10.1007/s00784015-1417-4

17. Sadr Haghighi AH, Pouyafar V, Navid A, Eskandarinezhad M, Abdollahzadeh Baghaei T. Investigation of the Optimal Design of Orthodontic Mini-Implants Based on the Primary Stability: A Finite Element Analysis. J Dent Res Dent Clin Dent Prospects (2019) 13:85-9. doi:10.15171/joddd.2019.013

18. Kravitz ND, Kusnoto B. Risks and Complications of Orthodontic Miniscrews. Am J Orthod Dentofacial Orthop (2007) 131(4 Suppl. 1):S43-51. doi:10.1016/ j.ajodo.2006.04.027

19. Chatzigianni A, Keilig L, Reimann S, Eliades T, Bourauel C. Effect of MiniImplant Length and Diameter on Primary Stability under Loading with Two Force Levels. Eur J Orthod (2011) 33:381-7. doi:10.1093/ejo/cjq088

20. Lakshmikantha HT, Ravichandran NK, Jeon M, Kim J, Park H-s. Assessment of Cortical Bone Microdamage Following Insertion of Microimplants Using Optical Coherence Tomography: a Preliminary Study. J Zhejiang Univ Sci B (2018) 19:818-28. doi:10.1631/jzus.b1700612

21. Seifi M, Matini NS. Evaluation of Primary Stability of Innovated Orthodontic Miniscrew System (STS): An Ex-Vivo Study. J Clin Exp Dent (2016) 8:e255-9. doi:10.4317/jced.52676

22. Lee NK, Baek SH. Effects of the Diameter and Shape of Orthodontic MiniImplants on Microdamage to the Cortical Bone. Am J Orthod Dentofacial Orthop (2010) 138:8-9. doi:10.1016/j.ajodo.2010.02.019

23. Aljekhedab F, Zhang W, Haugen HK, Wohl GR, El-Desouki MM, Fang Q. Influence of Environmental Conditions in Bovine Bone Ablation by Ultrafast Laser. J Biophotonics (2019) 12:e201800293. doi:10.1002/ jbio.201800293

24. Wu J, He J, Yin K, Zhu Z, Xiao S, Wu Z, et al. Robust Hierarchical Porous PTFE Film Fabricated via Femtosecond Laser for Self-Cleaning Passive Cooling. Nano Lett (2021) 21(10):4209-16. doi:10.1021/acs.nanolett.1c00038

25. Yin K, Chu D, Dong X, Wang C, Duan J-A, He J. Femtosecond Laser Induced Robust Periodic Nanoripple Structured Mesh for Highly Efficient Oil-Water Separation. Nanoscale (2017) 9(37):14229-35. doi:10.1039/c7nr04582d

Conflict of Interest: The authors declare that the research was conducted in the absence of any commercial or financial relationships that could be construed as a potential conflict of interest.

Publisher's Note: All claims expressed in this article are solely those of the authors and do not necessarily represent those of their affiliated organizations, or those of the publisher, the editors, and the reviewers. Any product that may be evaluated in this article, or claim that may be made by its manufacturer, is not guaranteed or endorsed by the publisher.

Copyright (c) 2021 Zhang, Alghannam, Zhu, Zhang, Wohl, Haugen, Qin, Xie, Fang and Shen. This is an open-access article distributed under the terms of the Creative Commons Attribution License (CC BY). The use, distribution or reproduction in other forums is permitted, provided the original author $(s)$ and the copyright owner(s) are credited and that the original publication in this journal is cited, in accordance with accepted academic practice. No use, distribution or reproduction is permitted which does not comply with these terms. 